\title{
Some biomedical topics with open problems of the University of Maryland microsystems laboratory research
}

\section{Background}

Under the direction of Professor N. DeClaris ${ }^{1}$ about 1967 the EE Department of the University of Maryland initiated biomedical engineering as one of the specialization areas for graduate study. This led to my transfer of the Microsystems Laboratory from Stanford to the University of Maryland where considerable concentration has been in the biomedical area. Some of the activities with open problems are listed below.

\section{VLSI neural networks}

One of the problems of hardware associated with biological neural behavior is to find efficient transistor circuits for making mimics by the thousands in Very Large Scale Integrated Circuits, VLSI. Among the programs that I started with Professor DeClairis ${ }^{1}$ was a joint one with Polish researchers under Professor Michal Bialko of Politechnika Gdanska which led to our neural type cell (NTC). ${ }^{2}$ This was initially fabricated using lumped circuits but has recently been redesigned for 0.35 micron fabrication ${ }^{3}$ and could be further reduced in size for upcoming technologies. Along with the NTC which behaves in its electrical behavior as the biological cell body, are the neural type junction (NTJ), which mimics the dendritic connections to the cell, and the neural type line (NTL) which mimics the biological axon; combined these allow one to make more elaborate neural type systems. Since the NTC designs depend upon an associated hysteresis and this depends upon the transistor parameters, it would be worth obtaining a design and fabrication using much smaller transistors, say 3 to 180 nanometer ones, along with sub-threshold designs. A number of other neural type systems have been investigated including that associated with locust flight ${ }^{4}$ which could be extended to control of new classes of drones based upon neural control.

\section{Osteoporosis}

Osteoporosis affects a very large number of people and consequently it is of interest to be able to accurately monitor its progress. For this purpose, with Professors Mona Zaghlul of George Washington University and Vicky Ikonomidou of George Mason University we have formed a group in which, with Professor Ikonomidou's students, it has been discovered that MRI scans can display osteoporosis progress. ${ }^{5}$ However, what is of interest is a portable detection system which can be used at home. For this my dentist, Dr. Mary Alexander, mentioned that her Harvard Medical Science doctoral dissertation indicates that the tooth may be an early indicator. Consequently, it should be possible to make a "tooth density indicator" that could be an excellent osteoporosis indicator for home use.

\section{Deep brain stimulation}

The use of Deep Brain Stimulators, DBS, for suppression of tremors in the later stages of Parkinson's disease is standard. But this involves drilling a hole in the brain, which would seem avoidable.
Volume 5 Issue I - 2019

Robert W Newcomb
ECE Department, University of Maryland, USA

Correspondence: Robert W Newcomb, ECE Department, University of Maryland, College Park, MD 20742, USA, Emailnewcomb@eng.umd.edu

Received: June 01, 2018 | Published: February 12, 2019

Thus, we began to look at the use of antennas on the scalp to achieve the same results "non-invasively." This led to the dissertation of Dr. Mohammad Safar, now of Kuwait University, upon which the paper ${ }^{6}$ is based. However, not much is theoretically known about the DBS mechanism. Consequently, recently with Professor Nevine El-Leithy the desire for mapping of the electromagnetic fields in the brain due to external magnetic fields, in cooperation with colleagues at the FDA, has led to a major undertaking by a large group of freshmen students (some 80 to date) via the First-year Innovation \& Research Experience, FIRE, program under the stream "Deep Brain Neurotech"7 In essence it is desired to obtain an external means of exciting the neurons which control various activities such as Parkinson's tremors. Preliminary results on low frequency excitation via coils on 3D printed helmets appear promising. In actual fact there appear to be a number of research groups investigating the electromagnetic fields in the brain for control of migraines, depression, epilepsy, Parkinson's, etc. The real challenge is to determine the field, and the affect it has, on an individual neuron of the kind located at a desired point in the brain and then find a means to excite this. Alternatively, excitations of other types, such as ultrasound via the throat or limbs seem possible topics for fruitful research.

\section{Ear type systems}

In an effort to model hearing behavior a program on ear-type systems was initiated with Professor Vcitoria Rodellar and Pedro Gomez at Universidad Politecnica de Madrid ${ }^{8}$ based on Kemp echoes which has been extended in various ways including research with Professor Louiza Sellami of the US Naval Academy ${ }^{9}$ and more recently in the research of Dr. Koranan Limpaphayom ${ }^{10}$ via data from helmets of the type mentioned above used for testing hearing of babies. This latter models the neural pathway that leads to ABM signals monitored via sensors on the brain. In turn this leads to the fascinating possibility of reversing the use of the sensors to insert signals into the brain as a type of hearing aid and possibly for the control of tinnitus. Possibly this could be done by use of the 3D helmets with standard embedded sensors.

\section{Some others}

Some other systems considered have to do with cancer elimination 
via use of transistor models for control of mitosis cell behavior, carried out with Professor Paul Sotiriadis of Athens University, ${ }^{11}$ where the bipolar transistor circuits covered could be extended to sub threshold CMOS ones. Another involves modeling of the heart for control of fibrillations, ${ }^{12}$ and a four-year undergraduate GEMSTONE project, CARDIO, ${ }^{13}$ is underway to use energy from the heart to charge pacemaker batteries. An alternate for this has been investigated by nearby high school students using solar power on hats with extensions possible to motion of the arms, legs, or throat. And a recent study has been of VLSI models of the hippocampus for control of motion. ${ }^{14}$

\section{Acknowledgments}

None.

\section{Conflicts of interest}

Author declares that there is no conflicts of interest.

\section{References}

1. De Claris N, Newcomb RW. The Circuit Imperative in Biomedical Engineering Education. Proceedings of the 8th Annual Northeast Bioengineering Conference. 1980:478-481.

2. Newcomb RW. Neural-Type Microsystems: Some Circuits and Considerations. Proceedings of the IEEE International Conference on Circuits and Computers (ICCC'80). 1980;2:1072-1074.

3. Joshua Rogers. Neural Type Cell. University of Maryland; 2017:19.

4. Ellen Curran Kelly Wooten. Design of a Coordinated Controller Based on a Neuron Model of Flight Locomotion in a Locust. 1997.
5. Anuraag Ravikumar, Elena Rantou, Vikas Kotari, et al. Osteoporosis detection from changes in cervical bone tecture analysis features on clinical brain MRI

6. Mohammad Safar, Robert W Newcomb. Design of a Hemispherical Antenna Array for Magnetic Field Control in the Brain. Procedings of the 2011 IEEE/NIH Life Science Systems Applications Workshop. 2011:131134.

7. FIRE DBM. Deep Brain Neurotech in The First-year Innovation and Research Experience.

8. Go'mez P, Rodellar V, Newcomb R. A PARCOR Characterization of the Ear for Hearing-Aids. Proceedings of the IEEE. 1982;70(12):1464-1466.

9. Sellami L, Newcomb RW. Synthesis of Kemp Echoes Using an Analog Choclea Circuit Model. Proceedings of CASME 2012. 2012;3-16.

10. Koranan Limpaphayom, Robert W Newcomb. Inverse Function Theory for Hearing Correction via the ABR. In: Bos L, Carroll D, editors. Future Visions on Biomedicine and Bioinformatics. Berlin: Springer; 2011:85-92.

11. Paul Sotiriadis, Robert W Newcomb. Model Reference Circuits for Mitosis Control. MED 09, 17th Mediterranean Conference on Control and Automation; 2009 June 25; Thessaloniki. 2009:564-569.

12. Li Wang. VLSI Design of Heart Model. 2007.

13. Class of 2019, Team CARDIO.

14. Anu Aggarwal. Neuromorphic VLSI Realization of the Hippocampal Formation and the Lateral Superior Olive. University of Maryland; 2015. 